\title{
Graphitization of SiC (0001) Surface in the Si Flux
}

\author{
Piotr Ciochoń, Jacek Kolodziej \\ Institute of Physics, Jagiellonian University \\ ul. Łojasiewicza 11, 30-348 Cracow, Poland \\ piotr.ciochon@doctoral.uj.edu.pl; jj.kolodziej@uj.edu.pl
}

\section{Extended Abstract}

Graphene, a two-dimensional allotrope of carbon has attracted great interest in the scientific, engineering and business communities in the past years, thanks to its extraordinary properties leading to numerous potential applications [1]. Despite recent advancements, synthesis of a large-scale, high-quality graphene remains challenging, especially in terms of transferring graphene monolayer on the insulating surface, required for future applications in electronics.

Surface graphitization of $\mathrm{SiC}(0001)$ in UHV via thermal annealing in the temperature $\mathrm{T}=1450 \mathrm{~K}$ has been proposed as a way to produce high quality graphene directly on an insulator. Method was improved by Emtsev et. al. [2] by performing annealing in the presence of buffer, inert gases with a pressure equal to around 1bar, leading to the slowed down sublimation of silicon atoms from the surface allowing for higher process temperatures and better organization of the carbon atoms in the graphene layers.

Because even purest buffer gasses lead to the substantial exposition of the surface to the contaminations compared to the UHV conditions, we have conducted graphitization process in the UHV, replacing the buffer gas with the ultra-pure flux of Si atoms from the external sublimation source, thus reducing the impurities concentration in the atmosphere several orders of magnitude. The source used in the experiments utilizes silicon sublimation from the solid filament and all source parts exposed to the vacuum are made from high purity monocrystalline silicon resulting in the stable, ultra-pure flux.

We have performed the graphitization of the SiC (0001) Si-terminated surfaces (4H polytype) in systematically varied conditions: annealing temperature, annealing time and the flux of $\mathrm{Si}$ atoms in order to achieve near-equilibrium graphitization conditions, which should result in the synthesis of a high-quality, uniform, large-scale monocrystalline graphene.

The results of the graphitization series were monitored using complimentary surface analysis techniques: low energy electron diffraction for the assessment of a large-scale crystallographic ordering and the presence of disordered regions, angle-resolved UV photoelectron spectroscopy for the measurement of the electronic properties of the synthesized graphene as well as the average number of graphene layers present on the surface and scanning tunnelling microscopy for the observation of the surface in the atomic scale and quantification of the size of monocrystalline domains.

We were able to optimize the process to achieve the formation of a graphene characterized by superior crystallographic ordering, large monocrystalline domains and high-quality electronic structure, characterized by n-type doping (Dirac point located around $0.4 \mathrm{eV}$ below Fermi level). ARPES measurements of the high-quality samples were performed at the newly opened SOLARIS synchrotron located in Kraków, Poland. The analysis of the data is ongoing and systematic trends linking the properties of synthesized graphene with the preparation conditions are expected to be observed and more detailed studies of the graphene grown in optimized conditions will be performed leading to the complete description of the process of the surface graphitization of $\mathrm{SiC}$ under $\mathrm{Si}$ flux.

\section{Acknowledgements}

We acknowledge financial support by National Science Centre Poland (contract 2014/15/N/ST5/00523) and by National Leading Research Centre „Matter-Energy-Future”. The research was carried out with the equipment purchased thanks to European Regional Development Fund in the framework of the Polish Innovation Economy Operational Program

\section{References}

[1] A. H. Castro Neto, et. al., "The electronic properties of graphene,” Rev. Mod. Phys., vol. 81, no. 1, pp. 109-162, 2009. 
[2] K. V. Emtsev, et. al., "Towards wafer-size graphene layers by atmospheric pressure graphitization of silicon carbide," Nature Materials, vol. 8, no. 3, pp. 203-207, 2009. 